\title{
Insulin therapy in the intensive care unit should be targeted to maintain blood glucose between $4.4 \mathrm{mmol} / \mathrm{l}$ and $6.1 \mathrm{mmol} / \mathrm{l}$
}

\author{
G. Van den Berghe
}

Received: 26 September 2007 / Accepted: 10 October 2007 / Published online: 27 November 2007

(C) Springer-Verlag 2007

Keywords Critical illness · Glucose $\cdot$ ICU · Intensive care · Insulin $\cdot$ Outcome $\cdot$ Randomised trials $\cdot$ Statistical power

\author{
Abbreviations \\ ARR absolute risk reduction \\ ITT intention-to-treat \\ ICU intensive care unit
}

Hyperglycaemia has been repeatedly associated with risk of mortality and morbidity in the intensive care unit (ICU). However, establishing a causal relationship between hyperglycaemia and adverse outcome requires randomised controlled trials assessing the impact of treating/preventing hyperglycaemia in this condition. The only two randomised controlled studies that have addressed this question so far targeted normoglycaemia (4.4-6.1 mmol/l) in ICUs and showed that the link indeed appears causal. The evidence currently available is thus in favour of a "normal $\leq 6.1 \mathrm{mmol} / \mathrm{l}$ ' level for blood glucose control in ICUs and is not supportive of J. Miles's viewpoint in this debate [1], as studies on any other level have not been performed.

The first randomised controlled trial from Leuven included adult patients admitted to ICU after extensive, complicated surgery or trauma, or after medical complications of major surgical procedures [2]. In the intervention group, glucose levels were targeted to $4.4-6.1 \mathrm{mmol} / 1$ with

G. Van den Berghe $(\bowtie)$

Department of Intensive Care Medicine,

Catholic University of Leuven,

Herestraat 49,

3000 Leuven, Belgium

e-mail: greet.vandenberghe@med.kuleuven.be a continuous intravenous insulin infusion, resulting in average blood glucose levels of $5.5 \mathrm{mmol} / 1$ (normoglycaemia). The control group was treated 'conventionally' (only insulin for hyperglycaemia $>11.2 \mathrm{mmol} / \mathrm{l}$ ) and had average blood glucose levels of $8.8 \mathrm{mmol} / \mathrm{l}$. At the start of the study, there were no data available on the size of the expected benefit, and thus interim analysis was performed for safety reasons. The study was stopped after inclusion of 1,548 patients. In the intention-to-treat (ITT) population, the intervention lowered ICU mortality rate from 8.0 to $4.6 \%$ [absolute risk reduction (ARR) 3.4\%] and in-hospital mortality rate from 10.9 to $7.2 \%$ (ARR 3.7\%). The benefit was larger in the target population of long-stay patients, with a reduction of ICU mortality rate from 20.2 to $10.6 \%$ (ARR 9.6\%) and of in-hospital mortality rate from 26.3 to $16.8 \%$ (ARR 9.5\%). In retrospect, it appeared indeed that the impact of the intervention increased with the duration of its application and that there was a substantial benefit clearly present from 3 days of intensive insulin therapy onwards. Besides saving lives, maintaining normoglycaemia prevented organ failure and shortened time on the ventilator and in the ICU. Maintaining normoglycaemia protected the central and peripheral nervous system and improved longterm rehabilitation of patients with brain injury [3], and evoked substantial cost-savings [4]. A 4 year follow-up of the cardiac surgery patients showed that it also improved long-term outcome with maintenance of the survival rate benefit without inducing additional need for medical care [5]. Subsequently, an observational study in a heterogeneous medical/surgical patient population $(n=1,600)$ confirmed the clinical and cost-saving impact of a tight glucose management protocol in 'real life' intensive care [6, 7].

Thereafter, two multi-centre randomised controlled trials were started, but stopped early. The first one was designed as a four-arm study to assess the impact of two types of 
fluid resuscitation and, concomitantly, of intensive insulin therapy in patients with severe sepsis and septic shock (VISEP) [8]. The insulin arm was stopped prematurely because the rate of hypoglycaemia (12.1\%) was considered too high. With data from only a very limited number of patients $(n=488), 90$ day mortality rate was $29.5 \%$ in the intensive vs $32.8 \%$ in the conventional arm (ARR 3.3\%, NS) [9]. The second trial stopped early was GLUCONTROL. This study was designed to investigate the external validity of the Leuven findings in a mixed population of ICU patients [10]. The safety committee decided to stop enrolment after a first interim analysis because the targeted level of glycaemic control was not reached, whereas $10 \%$ of the patients developed hypoglycaemia.

In Leuven, after the first surgical study, a second large randomised controlled trial was started in medical ICU patients using the same insulin titration protocol as in the previous surgical study [11]. Based on the outcome results of the surgical study, showing that the impact of the intervention depends on the duration of its application [2], the medical study was statistically powered to demonstrate or exclude an absolute $7 \%$ reduction of the risk of death among patients needing at least 3 days of intensive care (similar that observed in the surgical study). Long-stay patients cannot be identified upon admission, and thus, in order to obtain the required sample size of 700 long-stay patients, inclusion of 1,200 patients was considered necessary. Blood glucose was controlled to mean levels of $5.8 \mathrm{mmol} / 1$ in the intensive group compared with $8.9 \mathrm{mmol} / 1$ in the conventional group. In-hospital mortality rate of the ITT population of 1,200 patients was reduced from 40.0 to $37.3 \%$. The lack of statistical significance was not surprising as the study was not statistically powered for this endpoint. However, in the predetermined target group of long-stay patients, maintaining normoglycaemia significantly reduced in-hospital mortality rate from 52.5 to $43.0 \%$ (ARR $9.5 \%$ ). The morbidity rate was significantly reduced in the ITT group of patients, with less new kidney injury, less hyperbilirubinaemia, earlier weaning from mechanical ventilation, and earlier ICU and hospital discharge. The reduction in morbidity rate was even more striking in the target group of long-stay patients. These were discharged from the hospital alive on average 10 days earlier than on conventional insulin therapy. Among long-stay patients, intensive insulin therapy also reduced the incidence of critical illness polyneuropathy and/or myopathy [12].

The pooled data set of the two Leuven studies (mixed medical/surgical patient population; $n=2,748)[2,11]$ revealed that hospital mortality rate can be reduced from 23.6 to $20.4 \%$ (ARR $3.2 \%, p=0.04$ ) for all patients, and from 37.9 to $30.1 \%$ (ARR $7.8 \%, p=0.002$ ) in the patients who remained in the ICU for at least 3 days [13]. Objectively quantifiable morbidity endpoints, such as kidney injury developing during ICU stay and critical illness polyneuropathy, were reduced by one half.

Comparing all available studies reporting mortality rate data, it is striking to find a uniform $3-4 \%$ lower risk of death in ITT populations receiving intensive insulin therapy. Whether or not $3-4 \%$ ARR is statistically significant, of course, depends on statistical power to detect such a difference and thus on the size of the trial. Indeed, the size of a study required to assign a certain ARR as statistically significant depends: (1) on the baseline risk of death; (2) the size of the ARR that is anticipated. The higher the baseline risk and the smaller the anticipated ARR, the larger the sample size. Small studies, with only a few hundred patients, are unable to detect a 3-4\% ARR in mortality rate of ICU patients. To prove or exclude the effect shown in the Leuven studies with enough statistical power in ITT analysis of a multi-centre setting requires a sample size of at least 6,000 patients.

The impact of maintaining normoglycaemia increased with time in both the surgical and the medical Leuven studies. Indeed, a higher absolute reduction of mortality rate is present among long-stay patients (7-9\%) than in the ITT population (3-4\%), as the effect among long-stay patients is diluted by the lack of mortality benefit when applied only briefly. Pooling of the datasets from the surgical and the medical Leuven studies created the statistical power to show the morbidity and mortality benefits of intensive insulin therapy in the ITT mixed medical/surgical patient population [13]. Other studies are in agreement, with 3 days of glucose control being minimally required to obtain a sizable outcome benefit [14-16]. Furthermore, a surrogate endpoint, such as 30-day mortality rate, is inappropriate for a preventive strategy such as intensive insulin therapy, as clear separation of the survival rate curves occurs only after this time point. Small studies with 30-day mortality rate as endpoint are unable to provide evidence against the concept of tight glucose control in ICU.

For example, the VISEP study, stopped early for risk of hypoglycaemia after inclusion of only 488 patients, was clearly underpowered to assign the expected and documented $3-4 \%$ difference in mortality rate as statistically significant [8]. Furthermore, VISEP was a four-treatment-arm study (two fluid resuscitation strategies and two levels of blood glucose control) and 17 centres participated, which may have caused bias [8]. The GLUCONTROL trial, also stopped early for inadequate blood glucose control and risk of hypoglycaemia, has not published data yet on mortality rates, but it is clear that this study was also underpowered to exclude a $3-4 \%$ mortality rate benefit on only 855 patients from 19 centres [10].

Sceptics have suggested that the large Diabetes InsulinGlucose in Acute Myocardial Infarction (DIGAMI)-2, The Clinical Trial of Metabolic Modulation in Acute Myocar- 
dial Infarction Treatment Evaluation-Estudios Cardiologicos Latinoamerica (CREATE-ECLA) and Glucose Insulin in Stroke Trial (GIST) studies provide 'evidence against' intensive insulin therapy in ICU patients [17-19]. However, none of these studies achieved a difference in blood glucose and hence the critique is unjustified. What the studies do show is that short-term infusion of glucose with insulin and potassium without maintaining normoglycaemia does not work. Achieving the target of normoglycaemia does indeed appear crucial to bring about benefit of intensive insulin therapy. In clinical studies it is impossible to separate completely the impact of insulin infusion from that of blood glucose control, as they are done concomitantly. In contrast, in a rabbit model of prolonged critical illness, a four-arm study design (two normoglycaemic groups and two hyperglycaemic groups, each with either normal or elevated insulin levels) recently revealed that glycaemic control per se mediated the survival rate benefit of intensive insulin therapy, independent of insulin [20]. Mortality rate was $41.4 \%$ in hyperglycaemic versus $11.1 \%$ in normoglycaemic rabbits, whereas insulin levels did not contribute to the survival rate benefit.

The clinical data, although methodologically less suitable to answer this question for reasons mentioned above, are in line with the experimental observations. Indeed, in the surgical study, the risk of death appeared to be linearly correlated with the degree of hyperglycaemia, with no clear cutoff level below which there was no further benefit [21]. The highest risk of death was observed for the conventionally treated patients who developed severe hyperglycaemia (8.8-11.2 $\mathrm{mmol} / \mathrm{l})$, intermediate risk for patients who received conventional insulin therapy and who developed only moderate hyperglycaemia $(6.1-8.8 \mathrm{mmol} / \mathrm{l})$, whereas the lowest risk was present in the patients whose blood glucose levels were controlled at $\leq 6.1 \mathrm{mmol} / 1$ with intensive insulin therapy. This pattern of risk of death in relation to stratification of glycaemia was confirmed in the mixed medical/surgical patient population, with most benefit gained when glycaemia was controlled $\leq 6.1 \mathrm{mmol} / 1$ [12]. In patients with a history of diabetes mellitus prior to the critical illness, however, risk of death for the three strata of glucose control appeared to mirror this pattern, although no significant differences were present.

The level of glycaemic control also accounted for the morbidity rate benefits $[2,13,21]$. As for mortality rate, tight glycaemic control $\leq 6.1 \mathrm{mmol} / 1$ appeared to be of crucial importance for the prevention of critical illness polyneuropathy and kidney injury $[12,20]$. This underscores the importance of achieving tight glucose control within the normoglycaemic target range to obtain the clinical benefits. In the Leuven studies $[2,11,13], 70 \%$ of the patients allocated to intensive insulin therapy actually achieved a mean daily blood glucose level $\leq 6.1 \mathrm{mmol} / 1$. In contrast, at the time of interim analysis of the GLUCONTROL study, this was only $27 \%$ of the patients on intensive insulin therapy, whereas the incidence of hypoglycaemia was comparable $(10 \%)$ to the Leuven studies (11\%). If optimal level of blood glucose control, i.e. normoglycaemia, is not achieved and hypoglycaemia is frequent, the therapy is not likely to bring about benefit and thus only exposes patients to risks. Hyperglycaemia deleteriously affects cells that take up glucose passively, independent of insulin, including hepatocytes [22], alveolar cells, endothelial cells [20, 23], neurons [3] and immune cells [24]. Prevention of glucose toxicity to the mitochondrial compartment appears important [22]. However, insulin may also exert direct effects when hyperglycaemia is concomitantly avoided. These include partial correction of dyslipidaemia [25], prevention of excessive inflammation $[24,26]$ and attenuation of the cortisol response to critical illness [27].

What are the risks of targeting normoglycaemia (4.4$6.1 \mathrm{mmol} / \mathrm{l})$ ? The first and most feared one is hypoglycaemia, which, when severe and prolonged, may cause convulsions, coma and brain damage, as well as cardiac arrhythmias. The risk of hypoglycaemia (glucose $\leq 2.2 \mathrm{mmol} / \mathrm{l}$ ) with intensive insulin therapy increased from 0.8 to $5.1 \%$ in the surgical [2] and from 3.1 to $18.7 \%$ in the medical [11] ICU study. In particular, patients with sepsis appeared to be susceptible to the development of hypoglycaemia, with an overall incidence of $11.4 \%$ (3.0\% for conventional and 19.6\% for intensive insulin therapy) versus $3.9 \%$ for patients without sepsis $(1.2 \%$ for conventional and $6.8 \%$ for intensive insulin therapy) [28]. Importantly, these brief episodes of biochemical hypoglycaemia were not associated with obvious clinical problems. Indeed, hypoglycaemia did not cause early deaths, only minor immediate and transient morbidity was seen in a minority of patients, and no late neurological sequelae occurred among hospital survivors [13]. Nevertheless, it cannot be completely excluded that hypoglycaemia counteracted some of the survival rate benefit of intensive insulin therapy. Interestingly, however, in a recent nested casecontrol study, no causal link was found between hypoglycaemia in the ICU and death when case and control participants were matched for baseline risk factors and time in the ICU before the hypoglycaemic event [29]. These observations support the previous suggestion that hypoglycaemia in ICU patients who receive intensive insulin therapy may merely identify patients at high risk of dying rather then representing a risk on its own [30]. Clearly, the development of accurate, continuous blood glucose monitoring devices, and preferably closed-loop systems for computer-assisted blood glucose control in the ICU, will help to avoid hypoglycaemia.

The second possible risk of targeting normoglycaemia is that of hyperinsulinaemia. Multivariate logistic regression analysis had indeed identified the dose of insulin as a 
positive risk factor for mortality [2, 13, 21, 31]. Such an association between high insulin dose and mortality can either be explained by more severe insulin resistance in the sicker patients by a true deleterious effect of hyperinsulinaemia. It was recently shown that circulating insulin levels with intensive insulin therapy are only transiently higher than in conventionally treated patients, and that intensive insulin therapy actually improves insulin-sensitivity in the critically ill, possibly via its effect on blood glucose and lipids [25, 32].

The third possible risk that has been suggested is insulin therapy for less than 3 days. In the Leuven medical ICU study, among patients treated in ICU for $<3$ days, there were more deaths in the intensive $(56 / 209,26.8 \%)$ than in the conventional $(42 / 224,18.8 \%)$ group [10]. If these numbers reflect a true causal association, a harmful effect of intensive insulin therapy given briefly would be suggested, whereas beyond 3 days it becomes beneficial. However unlikely, this generated concern among practicing clinicians, as it is virtually impossible to predict which patient will require more than 3 days of intensive care. A post hoc exploratory mortality rate analysis revealed that this observation was explained by selection bias. Importantly, when correcting for the well-known upon-admission risk factors, the apparent difference in mortality rate disappeared. Furthermore, detailed analysis of the pooled datasets from the surgical and the medical ICU study showed, with enough statistical power, that brief insulin treatment for less than 3 days did not cause harm [13].

A final issue that has been raised as 'evidence against' is that intensive insulin therapy would merely antagonise deleterious effects of parenteral nutrition [33]. Nutritional guidelines were followed in the Leuven studies [2, 11, 34]. Enteral feeding was attempted as soon as possible when the patients were haemodynamically stable, but when the energy intake target could not be reached, parenteral feeding was given early to compensate for the deficit. Criticism has been raised that with this regimen patients were at risk of overfeeding, and that this regimen did not represent the approach adopted in many centres. It was suggested that intensive insulin therapy merely serves to offset risk associated with 'excessive' parenteral glucose. This important question was addressed in the analysis of the pooled dataset of the two Leuven studies, and the data argue against such criticism. Indeed, the benefit of intensive insulin therapy was independent of parenteral glucose load as the mortality rate was lowered both in the lowest and the highest tertile of parenteral glucose load in the ITT population and in all tertiles of parenteral feeding for patients treated in intensive care for at least 3 days [13]. The question of benefit of early parenteral feeding in ICU patients while maintaining normoglycaemia is an open one that is currently being investigated [35].
In conclusion, current evidence suggests that maintaining normoglycaemia (blood glucose $\leq 6.1 \mathrm{mmol} / 1$ and avoiding prolonged hypoglycaemia), reduces mortality and morbidity of critical illness. Confirmation of the 3$4 \%$ absolute reduction of mortality rate in an ITT analysis requires a sample size of 6,000 patients. Provided the target of normoglycaemia is reached in a large enough fraction of patients allocated to the intervention and overlap of blood glucose control with the control group is avoided, and provided excessive hypoglycaemia is prevented, such studies will generate the answers to the remaining questions.

Acknowledgements The work was supported by research grants from the KULeuven (GOA2007/14) and the Fund for Scientific Research (FWO), Flanders, Belgium (G.0533.06).

Duality of interest The author declares that there is no duality of interest associated with this manuscript.

\section{References}

1. Miles J, McMahon MM, Isley WL (2007) No, the glycaemic target in the critically ill should not be $\leq 6.1 \mathrm{mmol} / \mathrm{l}$. Diabetologia DOI 10.1007/s00125-007-0888-5

2. Van den Berghe G, Wouters P, Weekers F et al (2001) Intensive insulin therapy in critically ill patients. N Engl J Med 345:1359-1367

3. Van den Berghe G, Schoonheydt K, Becx P et al (2005) Insulin therapy protects the central and peripheral nervous system of intensive care patients. Neurology 64:1348-1353

4. Van den Berghe G, Wouters PJ, Kesteloot K et al (2006) Analysis of healthcare resource utilization with intensive insulin therapy in critically ill patients. Crit Care Med 34:612-616

5. Ingels C, Debaveye Y, Milants I et al (2006) Strict blood glucose control with insulin during intensive care after cardiac surgery: impact on 4-years survival, dependency on medical care and quality of life. Eur Heart J 27:2716-2724

6. Krinsley JS (2004) Effect of an intensive glucose management protocol on the mortality of critically ill adult patients. Mayo Clin Proc 79:992-1000

7. Krinsley JS, Jones RL (2006) Cost analysis of intensive glycemic control in critically ill adult patients. Chest 129:644-650

8. Brunkhorst FM, Kuhnt E, Engel C et al (2005) Intensive insulin therapy in patient with severe sepsis and septic shock is associated with an increased rate of hypoglycemia - results from a randomized multicenter study (VISEP). Infection 33 (Suppl 1):19 (abstract)

9. Kompetenznetz Sepsis. Available from http://webanae.med.unijena.de/WebObjects/DSGPortal.woa/WebServerResources/sepnet/ visep.html, accessed 22 December 2006

10. National Institutes of Health: Glucontrol study: comparing the effects of two glucose control regimens by insulin in intensive care unit patients. Available from http://www.clinicaltrials.gov/ct/ show/NCT00107601 and http://www.glucontrol.org/, accessed 22 December 2006

11. Van den Berghe G, Wilmer A, Hermans G et al (2006) Intensive insulin therapy in medical intensive care patients. N Engl J Med 354:449-461 
12. Hermans G, Wilmer A, Meersseman W et al (2007) Impact of intensive insulin therapy on neuromuscular complications and ventilator dependency in the medical intensive care unit. Am J Respir Crit Care Med 175:480-489

13. Van den Berghe G, Wilmer A, Milants I et al (2006) Intensive insulin therapy in mixed medical/surgical ICU: benefit versus harm. Diabetes 55:3151-3159

14. Furnary AP, Gao G, Grunkemeier GL et al (2003) Continuous insulin infusion reduces mortality in patients with diabetes undergoing coronary artery bypass grafting. J Thorac Cardiovasc Surg 125:1007-1021

15. Furnary AP, Wu Y (2006a) Clinical effects of hyperglycemia in the cardiac surgery population: the Portland Diabetic Project. Endocr Pract 12:22-26

16. Furnary AP, Wu Y (2006b) Eliminating the diabetic disadvantage: the Portland Diabetic Project. Semin Thorac Cardiovasc Surg 18:302-308

17. Malmberg K (1997) Prospective randomised study of intensive insulin treatment on long term survival after acute myocardial infarction in patients with diabetes mellitus. DIGAMI (Diabetes Mellitus, Insulin Glucose Infusion in Acute Myocardial Infarction) Study Group. BMJ 314:1512-1515

18. Malmberg K, Ryden L, Hamsten A, Herlitz J, Waldenstrom A, Wedel H (1996) Effects of insulin treatment on cause-specific oneyear mortality and morbidity in diabetic patients with acute myocardial infarction. DIGAMI Study Group. Diabetes InsulinGlucose in Acute Myocardial Infarction. Eur Heart J 17:1337-1344

19. Scott JF, Robinson GM, French JM et al (1999) Glucose potassium insulin infusions in the treatment of acute stroke patients with mild to moderate hyperglycemia: the Glucose Insulin in Stroke Trial (GIST). Stroke 30:793-799

20. Ellger B, Debaveye Y, Vanhorebeek I et al (2006) Survival benefits of intensive insulin therapy in critical illness. Impact of normoglycemia versus glycemia-independent actions of insulin. Diabetes 55:1096-1105

21. Van den Berghe G, Wouters PJ, Bouillon R et al (2003) Outcome benefit of intensive insulin therapy in the critically ill: insulin dose versus glycemic control. Crit Care Med 31:359-366

22. Vanhorebeek I, De Vos R, Mesotten D et al (2005) Strict blood glucose control with insulin in critically ill patients protects hepatocytic mitochondrial ultrastructure and function. Lancet 365:53-59
23. Langouche L, Vanhorebeek I, Vlasselaers D et al (2005) Intensive insulin therapy protects the endothelium of critically ill patients. J Clin Invest 115:2277-2286

24. Weekers F, Giuletti A-P, Michalaki M et al (2003) Endocrine and immune effects of stress hyperglycemia in a rabbit model of prolonged critical illness. Endocrinology 144:5329-5338

25. Mesotten D, Swinnen JV, Vanderhoydonc F et al (2004) Contribution of circulating lipids to the improved outcome of critical illness by glycemic control with intensive insulin therapy. J Clin Endocrinol Metab 89:219-226

26. Hansen TK, Thiel S, Wouters PJ et al (2003) Intensive insulin therapy exerts anti-inflammatory effects in critically ill patients, as indicated by circulating mannose-binding lectin and C-reactive protein levels. J Clin Endocrinol Metab 88:1082-1088

27. Vanhorebeek I, Peeters RP, Vander Perre S et al (2006) Cortisol response to critical illness: effect of intensive insulin therapy. J Clin Endocrinol Metab 91:3803-3813

28. Van Cromphaut S, Wilmer A, Van den Berghe G (2007) Management of sepsis. N Engl J Med 356:1179-1181; author reply $1181-1182$

29. Vriesendorp TM, DeVries JH, van Santen S et al (2006) Evaluation of short-term consequences of hypoglycemia in an intensive care unit. Crit Care Med 34:26714-2718

30. Mackenzie I, Ingle S, Zaidi S et al (2006) Hypoglycemia? So what! Intensive Care Med 32:620-621

31. Finney SJ, Zekveld C, Elia A et al (2003) Glucose control and mortality in critically ill patients. J Am Med Assoc 290:2041-2047

32. Langouche L, Vander Perre S, Wouters P, D'Hoore A, Hansen TK, Van den Berghe G (2007) Effect of intensive insulin therapy on insulin sensitivity in the critically ill. J Clin Endocrinol Metab 92:3890-3897

33. Malhotra A (2006) Intensive insulin in intensive care. $\mathrm{N}$ Engl J Med 354:516-518

34. Jolliet P, Pichard C, Biolog G et al (1998) Enteral nutrition in intensive care patients: a practical approach. Intensive Care Med 24:848-859

35. Impact of early parenteral nutrition completing enteral nutrition in adult critically ill patients (EPaNIC study-ClinicalTrials.gov Identifier: NCT00512122), available at http://clinicaltrials.gov/ct/ show/NCT00512122, accessed 24 September 2007 